\title{
直接活化芳香 $\mathrm{C}-\mathrm{H}$ 键构建芳香 $\mathrm{C}-\mathrm{N}$ 键的研究进展
}

\author{
徐娟魏 真李加荣* \\ (北京理工大学化工与环境学院 北京 100081)
}

\begin{abstract}
摘要 芳香 $\mathrm{C}-\mathrm{N}$ 键广泛存在于药物、活性天然产物和农药中, 其构建方法是目前合成热点之一. 芳香 $\mathrm{C}-\mathrm{H}$ 键到芳香 $\mathrm{C}-\mathrm{N}$ 键的转化是重要的有机转化类型, 这种转化是构成芳胺的一种高效方法. 这种直接转化具有原子经济性, 可持续 发展性以及环境友好的特点. 主要以芳香 $\mathrm{C}-\mathrm{H}$ 键的氮宾插入和氧化胺化为主线, 综述了近十年来该领域的研究进展. 关键词 芳香 $\mathrm{C}-\mathrm{H}$ 键的活化; 胺化; 芳胺; 氮宾插入; 氧化胺化
\end{abstract}

\section{Research Progress in the Construction of Aromatic $\mathrm{C}-\mathrm{N}$ Bond from Activation of Aromatic $\mathrm{C}-\mathrm{H}$ Bond}

\author{
Xu, Juan Wei, Zhen Li, Jiarong* \\ (School of Chemical Engineering and Environment, Beijing Institute of Technology, Beijing 100081)
}

\begin{abstract}
Amines are important compounds that are found throughout the pharmaceutical, bioactive natural products and agrochemical industries. The transformation from aromatic $\mathrm{C}-\mathrm{H}$ bond to aromatic $\mathrm{C}-\mathrm{N}$ bond is an important organic conversion. It is an effective method for the construction of aromatic amine. This transformation has the characteristic of atom economy, sustainable development and environment friendly. This review is focused on the research progress in the direct amination of $\mathrm{C}-\mathrm{H}$ bonds in the past decade.
\end{abstract}

Keywords activation of aromatic $\mathrm{C}-\mathrm{H}$ bond; amination; aromatic amine; nitrene insertion; oxidative amination

芳胺是重要的有机化工原料, 广泛存在于染料、医 药、农药、表面活性剂、纺织助剂、螯合剂和高分子材 料等领域 ${ }^{[1]}$. 近年来, 构建芳香 $\mathrm{C}-\mathrm{N}$ 键的方法有了长 足的进展, 传统的经由硝化、还原等经典方法已逐渐为 芳香卤代物、三氟甲磺酸芳香酯、芳香硼化物和芳香硅 化物的催化胺化所取代 ${ }^{[2 \sim 4]}$, 如 Buchwald-Hartwig 交叉 偶联以及各种改进的 Ullmann 反应等 ${ }^{[5 \sim 8]}$. 比较而言, 在化合物中直接将芳香 $\mathrm{C}-\mathrm{H}$ 键转化成 $\mathrm{C}-\mathrm{N}$ 键已日渐 成为一种构建芳胺的十分有效的方法 ${ }^{[9 \sim 12]}$. 这种方法由 于是芳香 $\mathrm{C}-\mathrm{H}$ 键直接到芳香 $\mathrm{C}-\mathrm{N}$ 键的转化而不涉及 其它官能团, 因此是原子经济的, 能满足可持续发展和 环境友好的需求, 已成为芳胺合成的主要发展方向. 目 前由芳香 $\mathrm{C}-\mathrm{H}$ 键直接转化成芳香 $\mathrm{C}-\mathrm{N}$ 键的常用策略 有两种: 一种是由氮宾直接插入芳香 $\mathrm{C}-\mathrm{H}$ 键中 (Strategy A in Scheme 1), 另一种是芳烃的氧化胺化 (Strategy B in Scheme 1) ${ }^{[13]}$. 前者需要活化的氮宾中间 体, 后者需要引入氧化剂, 两者都需要高效的催化剂 (5\% 20\%). 本文对近十年来直接活化芳香 $\mathrm{C}-\mathrm{H}$ 键生
成芳胺的转化作一综述.

$$
\text { catalyst }
$$

Scheme 1

\section{1 氮宾插入}

金属催化下的氮宾插入是实现芳香 $\mathrm{C}-\mathrm{H}$ 键到芳香 $\mathrm{C}-\mathrm{N}$ 键转化的主要方法之一. 这种方法中一般使用的 金属催化剂包括 $\mathrm{Pd}, \mathrm{Co}, \mathrm{Fe}, \mathrm{Rh}, \mathrm{Ru}, \mathrm{Mn}, \mathrm{Cu}, \mathrm{Ag}, \mathrm{Ir}$ 等 ${ }^{[14 ~ 22]}$. 这种胺化的机理如 Scheme 2 所示. 即金属催 化剂 1 活化并插入芳香 $\mathrm{C}-\mathrm{H}$ 键, 氧化加成形成中间体 2,2 与胺形成新的配合物 3,3 去质子生成 4,4 还原消除 催化配体生成目标物芳胺, 再生的催化剂进入下一个循

*E-mail: jrli@bit.edu.cn

Received August 12, 2011; revised January 11, 2012; accepted February 8, 2012. 
环.

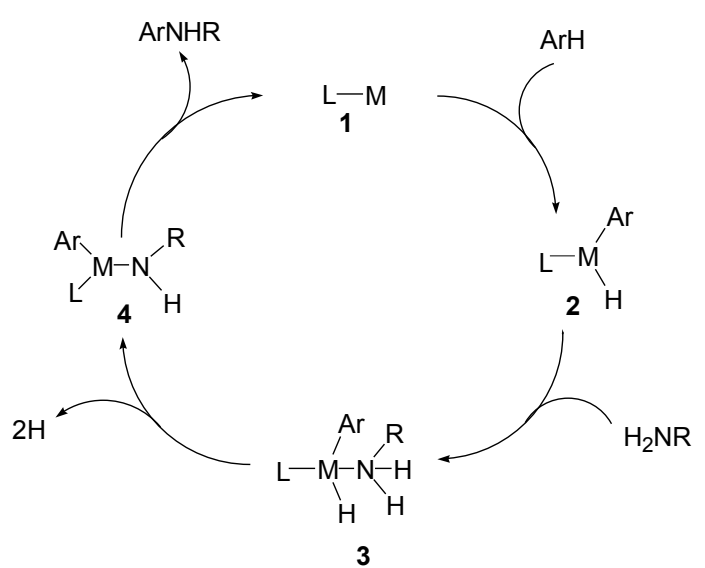

Scheme 2

\section{1 分子内氮宾插入胺化}

铑是催化芳香 $\mathrm{C}-\mathrm{H}$ 键活化的有效试剂. 2007 年, Driver 等 ${ }^{[23]}$ 发现一种铑诱导的芳香 $\mathrm{C}-\mathrm{H}$ 键的胺化, 可 将芳香乙烯基叠氮化合物变成吲哚或者其他含氮杂环 化合物(Scheme 3). 该反应对于芳环上含有供电子和吸 电子的取代基都有良好的效果. 尽管 1 位和 2 位取代的 萗乙烯基叠氮都能转化成吲哚, 但 2 位取代需要稍微苛 刻一点的条件; 2 位取代的苯并呋喃、呋喃和噻吩也都能 有效转化.

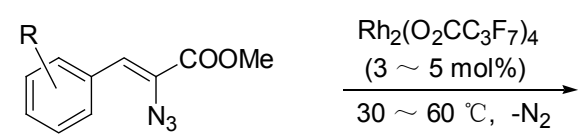

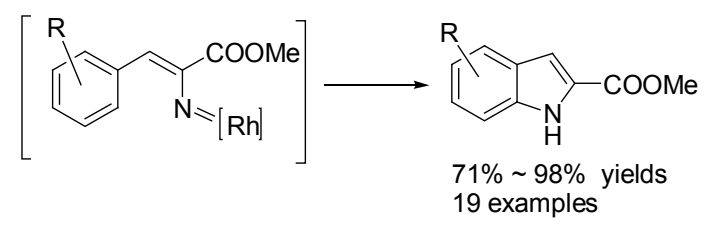

Scheme 3

同年, Narasaka 等 ${ }^{[24]}$ 报道了三氟乙酸铑催化 2-甲基3,3-二芳基- $1 H$ 氮杂丙烯啶异构化发生芳环 $\mathrm{C}-\mathrm{H}$ 键到 芳香 $\mathrm{C}-\mathrm{N}$ 键的转化, 得到 2,3-二取代的吲哚(Eq. 1). 该 方法可成功应用于 2 位含有氧基等强吸电子取代的吲哚 衍生物的合成. 而该类化合物很难由已知的常规方法制 得.

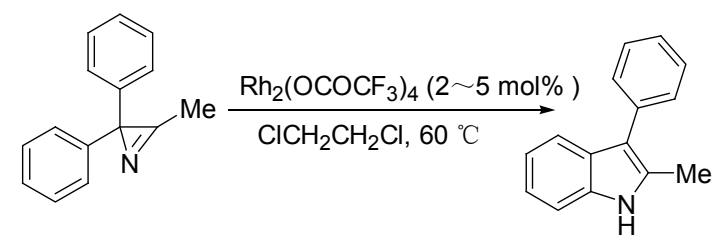

钯也是氮宾插入胺化的常用催化剂. 2010 年, Hart- wig 等 ${ }^{[13]}$ 报道了钯(II)催化的芳香 $\mathrm{C}-\mathrm{H}$ 键的胺化，可将 肟酯转化为吲哚化合物(Eq. 2). 结果显示, 吸电子取代 基对 $\mathrm{C}-\mathrm{H}$ 胺化的影响很小; 而给电子取代基不利于该 转化; 对于芳香氯化物和溴化物而言, 钯(0)优先与醋酸 肜的氮氧键反应, 而不是碳卤键, 因而具有一定的选择 性.

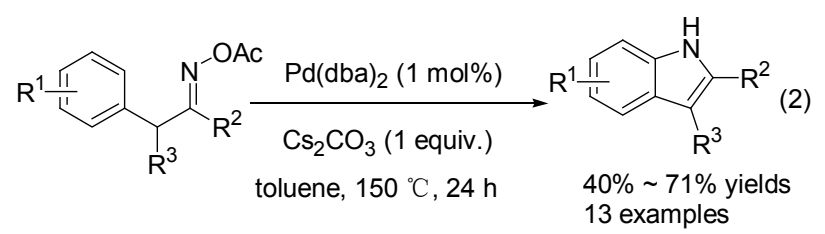

含有五氟苯甲酰基的肪酯与计量的 $\operatorname{Pd}\left(\mathrm{PCy}_{3}\right)_{2}$ 作用 能以很高的产率得到 $\operatorname{Pd}(\mathrm{II})$ 复合物(Scheme 4), 其结构 已由 $X$ 衍射得到证实. 该复合物是第一个分离得到的钯 配位的活性中间体.

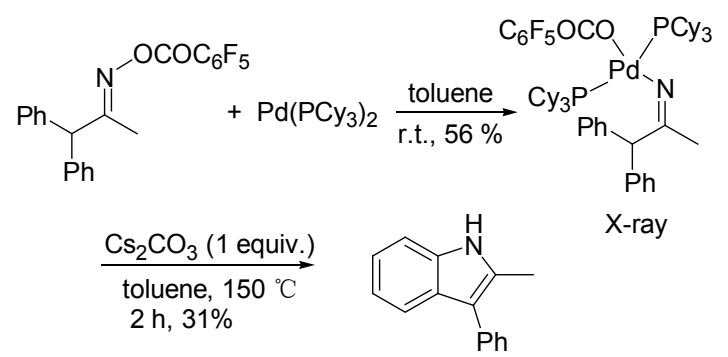

\section{Scheme 4}

\section{2 分子间氮宾插入胺化}

2003 年, Pe'rez 等 ${ }^{[25,26]}$ 研究发现铜(I)-单蝎型配合物 能催化活化芳香 $\mathrm{C}-\mathrm{H}$ 键并使之胺化, 且证实 $\mathrm{Tp}^{\mathrm{Br} 3} \mathrm{Cu}(\mathrm{NCMe})$ 具有最好的催化效果(Eq. 3). 在催化量 的 $\mathrm{Tp}^{\mathrm{Br} 3} \mathrm{Cu}(\mathrm{NCMe})$ 存在下, $\mathrm{PhI}=\mathrm{NTs}$ 与苯反应得到 40 ： 60 的 PhNHTs 和 $\mathrm{TsNH}_{2}{ }^{[27]}$.

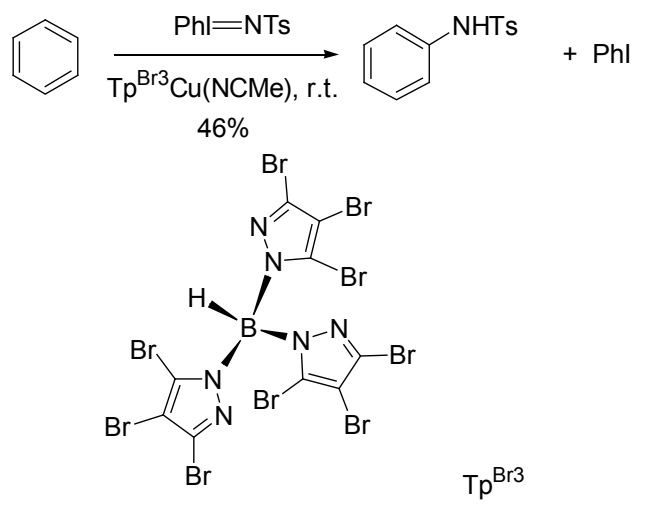

2007 年, $\mathrm{He}$ 等 ${ }^{[28]}$ 报道了室温下金催化氮宾插入惰 性芳香 $\mathrm{C}-\mathrm{H}$ 键的反应(Eq. 4), 三取代及更多取代的苯 在 1 $2 \mathrm{~mol}^{\circ} \mathrm{AuCl}_{3}$ 的催化下能发生转化. 但取代基比 较少的芳香物以及苯自身在该条件下反应的产率小于 
$5 \%$.<smiles>Cc1cc(C)cc(C)c1</smiles>

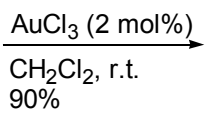<smiles>Cc1cc(C)c(NS(=O)(=O)[O-])c(C)c1</smiles>

同年, Sanford 等 ${ }^{[29,30]}$ 报道了钯(II)催化下的氮杂菲 10 位胺化(Scheme 5), 这里, 钯还通过与氮配位发挥定 位效应。

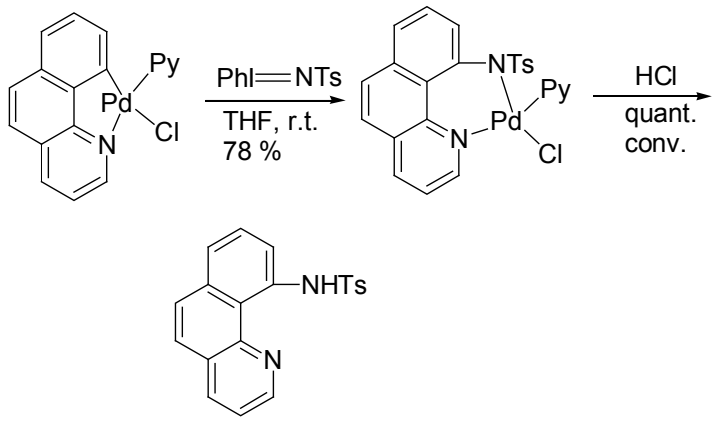

Scheme 5

杂环化合物与铁的复合物也可以有效诱导分子内 的氮宾转移反应 ${ }^{[31]} .2009$ 年, Betley 等 ${ }^{[32]}$ 报道了二吡咯 亚甲基-铁复合物能使有机叠氮化合物进行氮宾插入而 形成胺(Scheme 6).

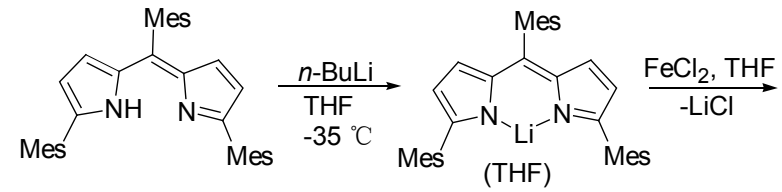

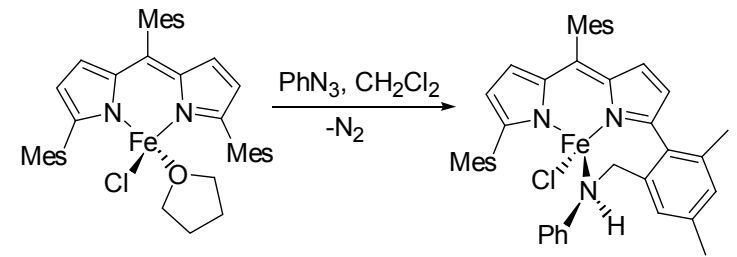

\section{Scheme 6}

2010 年, $\mathrm{Yu}$ 等 ${ }^{[33]}$ 发现氨基甲酸酯可以作为酰芳胺 的有效胺化试剂. 这里, 酰胺基作为芳香 $\mathrm{C}-\mathrm{H}$ 键胺化 的邻位导向基团. 此方法条件相对温和, 具有很好的高 活性基团耐受性、区域选择性和较宽的底物适用范围 (Eq. 5).

\section{2 氧化胺化}

氧化胺化是除氮宾插入外, 另一种很重要的直接合 成芳香胺的方法 ${ }^{[34 ~ 37]}$. 氧化胺化是指在芳香 $\mathrm{C}-\mathrm{H}$ 键直 接活化构建芳香 $\mathrm{C}-\mathrm{N}$ 键的体系中加入氧化剂, 氧化剂

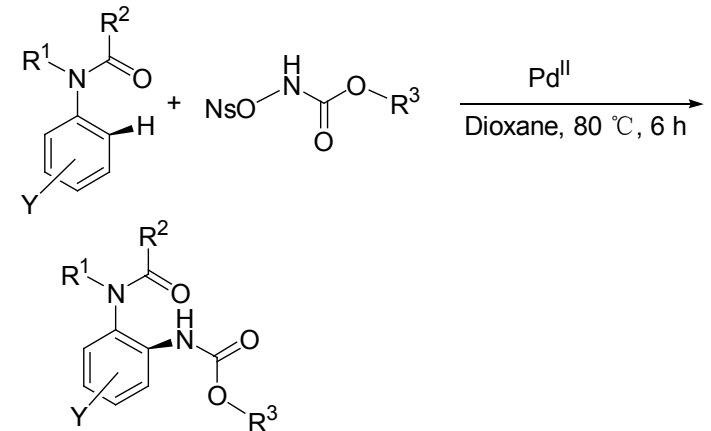

$$
\begin{array}{ll}
45 \% \sim 87 \% \text { yields } & \mathrm{Y}=\mathrm{F}, \mathrm{Cl}, \mathrm{Br}, \mathrm{OMe}, \text { OPiv, benzyl, vinyl } \\
21 \text { examples } & \mathrm{R}^{1}=\mathrm{H}, \text { Alkyl } \\
& \mathrm{R}^{2}=t-\mathrm{Bu}, \mathrm{Me}, \mathrm{Ph} \\
& \mathrm{R}^{3}=\mathrm{Et} \text {, benzyl, } \mathrm{CH}_{2} \mathrm{CCl}_{3}
\end{array}
$$

的加入十分有利于芳氢的失去. 此过程涉及催化剂体系 的耖选、胺化剂的选择和氧化剂的选择.氧化胺化分为两 种情况: 分子内氧化胺化和分子间氧化胺化. 分子内的 氧化胺化通常形成咔唑、吲唑等含氮杂环化合物.

\section{1 分子内氧化胺化}

2004 年, Matsubara 等 ${ }^{[38 ~ 40]}$ 报道了液压热水作为氧 化剂、铂催化芳香 $\mathrm{C}-\mathrm{H}$ 键活化生成咔唑的反应(Eq. 6).

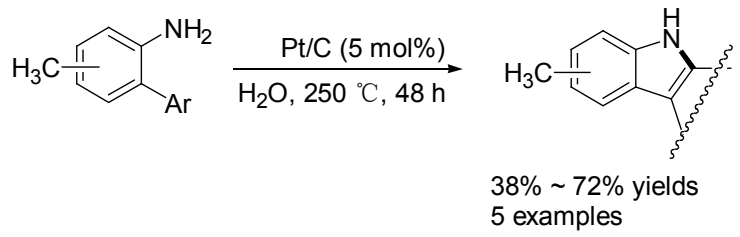

2005 年, Buchwald 等 ${ }^{[41]}$ 报道了醋酸钯催化、醋酸铜 和氧气共同作用下的芳香 $\mathrm{C}-\mathrm{H}$ 键到芳香 $\mathrm{C}-\mathrm{N}$ 键的转 化，这种转化可用于制备简单的非对称咔唑衍生物(Eq. 7).

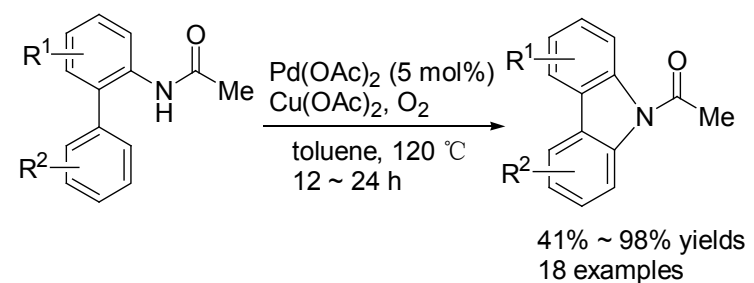

这里, $\mathrm{C}-\mathrm{H}$ 键所在的芳环能兼容多种吸电子和给 电子基团，但取代基的存在会影响 $\mathrm{C}-\mathrm{H}$ 键活化的选择 性，一般选择空间位阻较小的 $\mathrm{C}-\mathrm{H}$ 键活化. 可能的反 应历程如 Scheme 7. 底物的酰胺与醋酸钯进行配位, 然 后活化相连芳环的邻位 $\mathrm{C}-\mathrm{H}$ 键形成六元钯配合物，该 中间体随后还原消除得到产品和钯 $(0)$ 化合物. 钯 $(0)$ 被 醋酸铜重新氧化成钯(II), 还原后的铜(I)被氧气氧化成 铜(II), 从而完成催化循环. 


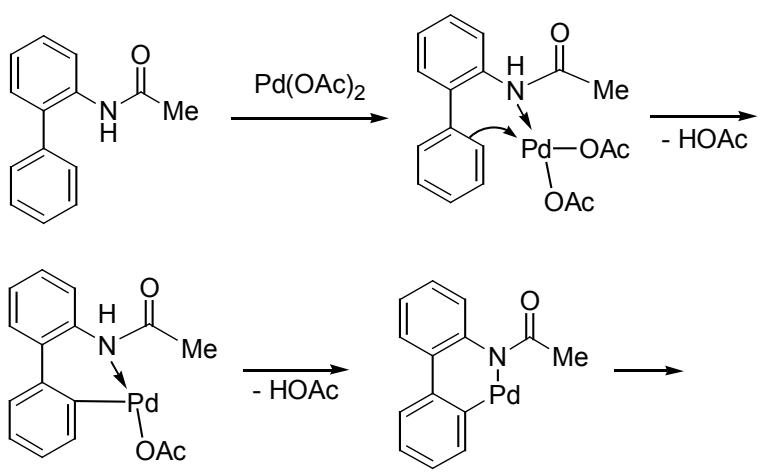

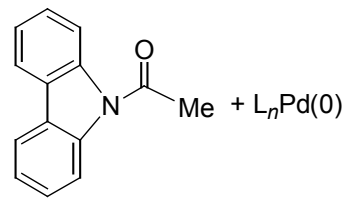

Scheme 7

随后他们 ${ }^{[22]}$ 发现二甲基亚砜可以替代醋酸铜从而 增加官能团兼容性, 扩大反应的底物范围(Eq. 8). 例如, 在 $120{ }^{\circ} \mathrm{C}$, 双芳环上分别含有吸电子的酰基、酯基、硝 基和氧基及推电子基团都能够合成咔唑. 该方法可以有 效合成非对称咔唑, 是已知合成咔唑类生物碱方法的补 充.

$$
\begin{aligned}
& \text { is molecular sieves } \\
& 78 \% \sim 94 \% \text { yields } \\
& 10 \text { examples } \\
& \mathrm{R}^{1}=\mathrm{H}, \mathrm{Me} \text {, OMe, } \mathrm{F}, \mathrm{CF}_{3}, \mathrm{COOMe} \\
& \mathrm{R}^{2}=\mathrm{H}, \mathrm{Me}, \mathrm{OMe}, \mathrm{F}, \mathrm{CF}_{3}, \mathrm{C}(\mathrm{O}) \mathrm{Me} \text {, COOMe, } \\
& \mathrm{SMe}, \mathrm{NO}_{2}, \mathrm{CN} \text {, OTIPS, TBS }
\end{aligned}
$$

2005 年, Yu 等 ${ }^{[3 \sim}$-46]发展了一种碘乙酸和铜、钯共 作用下的芳香 $\mathrm{C}-\mathrm{H}$ 键活化的新策略, 即从芳基取代的 脂肪胺直接制备二氢吲哚、四氢异喹啉(Eq. 9). 用此方 法可以使一些天然的氨基酸如苯基丙氨酸、酪氨酸和色 氨酸等转化为不同的杂环. 目前这种方法应用于医药杂 环化合物库的合成.

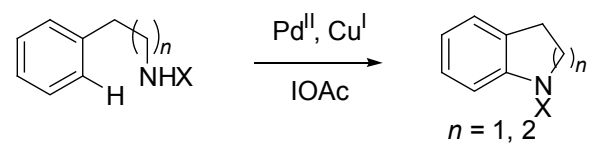

2007 年, Inamoto 等 ${ }^{[47]}$ 报道了醋酸铜和三氟乙酸银 存在下、钯(II)活化二芳基腙的芳香 $\mathrm{C}-\mathrm{H}$ 随后进行分子 内胺化合成吡唑的方法(Eq. 10). 底物的两个芳环上如 都在间位有推电子的甲氧基能极大地加速这个转化过 程, 定量产生高区域选择性的产物. 有意思的是, 这两
个取代基如果不同(如甲氧基和其他基团)，关环只发生 在甲氧基取代的富电子的芳环上，表明此环化过程同时 受到芳烃的空间和电子效应的影响.

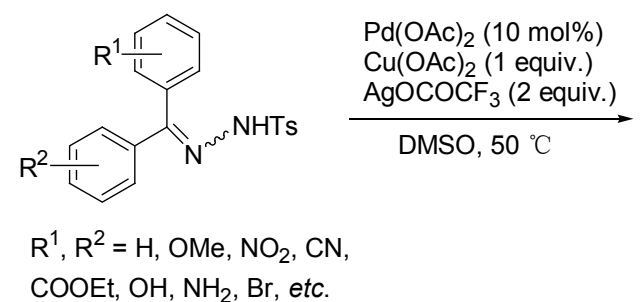

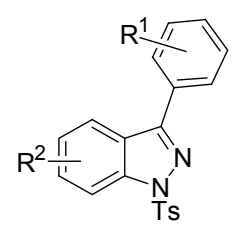

and/or<smiles>[R]CC(C)Cc1nn(S)c2c1C=C[R1]C=C2</smiles>

$13 \% \sim 99 \%$ yields 16 examples
2008 年, $\mathrm{Yu}$ 等 ${ }^{[48,49]}$ 报道了醋酸银、氯化亚铜及钯共 同作用下分子内的 $\mathrm{C}-\mathrm{H}$ 键氧化内酰胺化可制备 $\beta-\gamma-$ 和 $\delta$-内酰胺(Eq. 11).
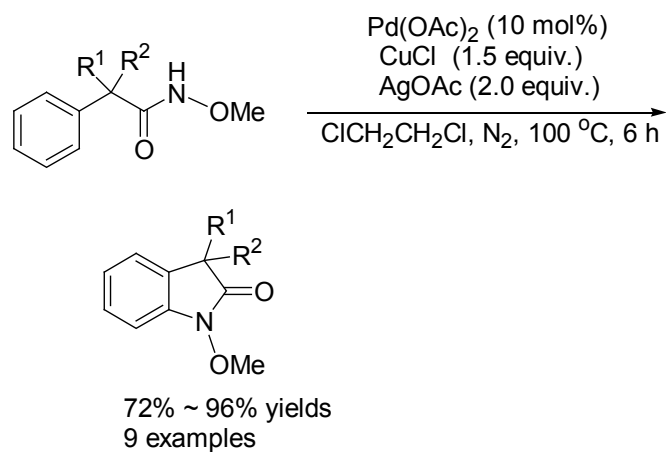

同年, Gaunt 等 ${ }^{[50]}$ 报道了二乙酸碘苯氧化、钯(II)催 化芳香 $\mathrm{C}-\mathrm{H}$ 键胺化形成咔唑的方法(Eq. 12). 这里，芳 环的取代基具有较好的耐受性. 更重要的是, 碘取代的 衍生物以较高产率顺利生成咔唑, 不会受钯的影响而断 开 $\mathrm{C}-\mathrm{I}$ 键.

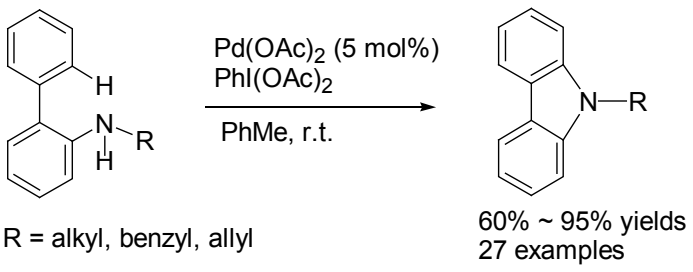

与此同时, Buchwald 等 ${ }^{[51]}$ 报道了以氧气为氧化剂、 醋酸铜催化芳基脒的芳香 $\mathrm{C}-\mathrm{H}$ 键活化到 $\mathrm{C}-\mathrm{N}$ 键的转 化方便地合成苯并咪唑衍生物(Eq. 13). 芳香环上含有 卤素、供电子或吸电子取代基时都具有较好的兼容性. 
其中, 生成苯并咪唑的 5,6 位上有取代时, 产率较高; 而产物的 4 位或者 4, 6 位上有双取代时, 产率较低. 该 方法可扩展制备 $N$-甲基化的 2-苯基苯并咪唑和 $N$-芳基 化苯并咪唑.

$$
\begin{aligned}
& \underset{\substack{\mathrm{DMSO}, 100^{\circ} \mathrm{C} \\
\mathrm{O}_{2}, 18 \mathrm{~h}}}{\mathrm{HOAc}(5 \text { equiv. })} \\
& 68 \% \sim 89 \% \text { yields } \\
& 17 \text { examples }
\end{aligned}
$$

随后, Inamoto 等 ${ }^{[52]}$ 报道了钯催化下芳香 $\mathrm{C}-\mathrm{H}$ 键的 氧化胺化, 即从烯胺化合物直接生成吲哚(Eq. 14). 虽然 该方法产率一般, 但显示了很好的原子经济性和快捷合 成, 可以与以前报道的方法相互结合获得各种吲哚衍生 物.

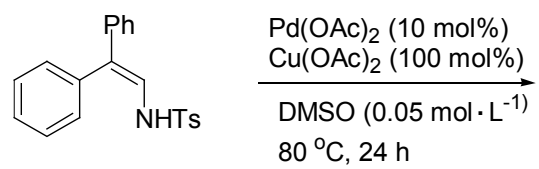<smiles>Sn1cc(-c2ccccc2)c2ccccc21</smiles>

2009 年, $\mathrm{Yu}$ 等 ${ }^{[53]}$ 发展了硫酸铈或氟鎓离子(三氟甲 磺酰酸 $N$-氟-2,4,6-三甲基吡啶鎓盐)作用下、醋酸钯催化 芳基脂肪胺环化形成二氢吲哚衍生物. 氟鎓盐是此胺化 反应非常有效的氧化剂, 能够广泛拓展底物范围(Eq. 15). 含有强吸电子基团(如卤素、乙酰基、氭基和硝基 等)的底物也可进行该反应; 间位有取代时展现出良好 的区域选择性，即选择较小空间位阻处形成碳氮键.
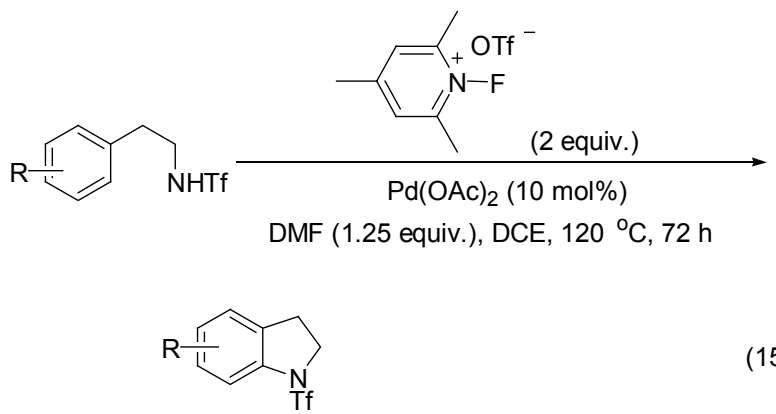

$28 \% \sim 91 \%$ yields 24 examples

2011 年, Youn 等 ${ }^{[54]}$ 开发了过硫酸氢钾为氧化剂、醋 酸钯催化的分子内芳香 $\mathrm{C}-\mathrm{H}$ 键的氧化胺化, 由 2-芳基 苯胺制备咔坐(Scheme 8). 该反应条件温和, 且过硫酸 氢钾廉价安全易于处理，显示出良好的普适性.

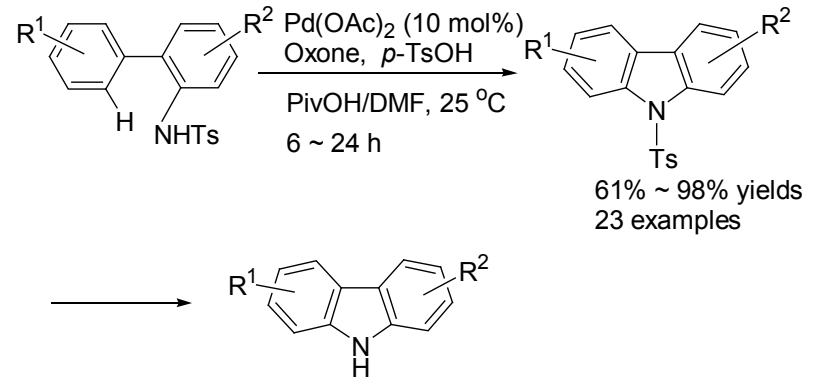

Scheme 8

\section{2 分子间氧化胺化}

氨基铜是较强的亲核胺化试剂, 2006 年 Knochel 等 ${ }^{[1]}$ 发现其与氯醌组合能有效地进行芳香 $\mathrm{C}-\mathrm{H}$ 键的胺 化，该反应的底物中即使含有卤素取代基也不发生卤素 的取代胺化(Scheme 9).<smiles>CC(c1cccc(N(c2ccc(Cl)cc2)c2cc(I)cc(C(F)(F)F)c2)c1)(C(F)(F)F)C(F)(F)F</smiles>

Scheme 9

近年来，他们 ${ }^{[55]}$ 发展了一种新的有效的多金属有 机胺组合试剂，该试剂是四甲基哌啶与氯化镁、氯化锂 等的结合体, 可用于活化各种 $\mathrm{C}-\mathrm{H}$ 键构建 $\mathrm{C}-\mathrm{C}$ 键和 $\mathrm{C}-\mathrm{N}$ 键, 且显示出独特的化学选择性和反应性(Eq. 16).

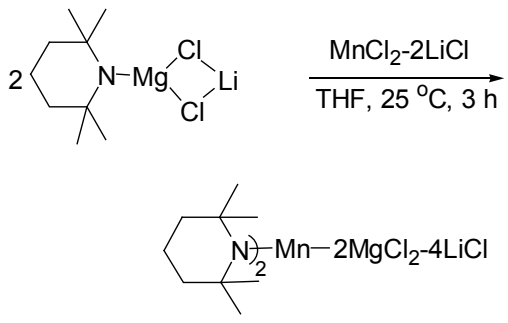

他们 ${ }^{[56]}$ 还发现，在类似的铜、锂、胺体系中引入醋 酸碘苯可方便地实施由锌活化的杂芳香 $\mathrm{C}-\mathrm{H}$ 键, 并最 终得到杂芳胺(Eq. 17). 


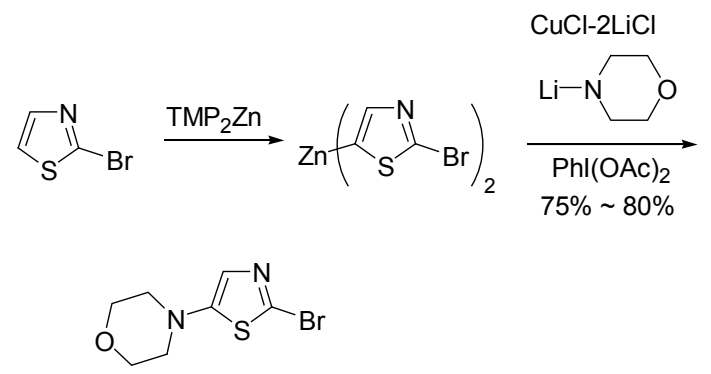

2006 年, $\mathrm{Yu}$ 等 ${ }^{[57]}$ 发现 $\mathrm{TsNH}_{2}$ 可以作为铜(II)催化芳 香 $\mathrm{C}-\mathrm{H}$ 键氧化胺化的氮负离子源(Eq. 18). 这里, 2-吡 啶基起到邻位导向基团的作用.

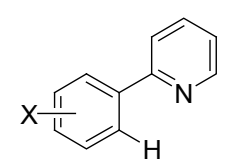

$$
\begin{aligned}
& \mathrm{Cu}(\mathrm{OAc})_{2}, \mathrm{O}_{2} \\
& \text { Nucleophiles }\left(\mathrm{Nu}^{-}\right) \\
& \stackrel{\text { Solvent, } 1300^{\circ} \mathrm{C}}{\longrightarrow} \\
& 40 \% \sim 90 \%
\end{aligned}
$$

$\mathrm{X}=\mathrm{H}, \mathrm{Me}, \mathrm{F}, \mathrm{Cl}, \mathrm{CF}_{3}, \mathrm{OMe}$, COOMe, $\mathrm{CHO}, \mathrm{CH}=\mathrm{CH}_{2}$

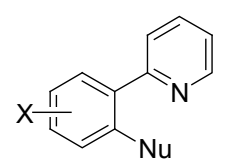

$\mathrm{Nu}=\mathrm{OH}, \mathrm{OAc}, \mathrm{Cl}, \mathrm{Br}, \mathrm{I}$, $\mathrm{TsNH}, \mathrm{PhS}, \mathrm{MeS}, \mathrm{CN}$

同年, Che 等 ${ }^{[58]}$ 采用类似的原理, 将氧化剂改用更 强的焦硫酸钾, 成功地在芳环的 2-位引入酰胺基团 (Eq. 19).

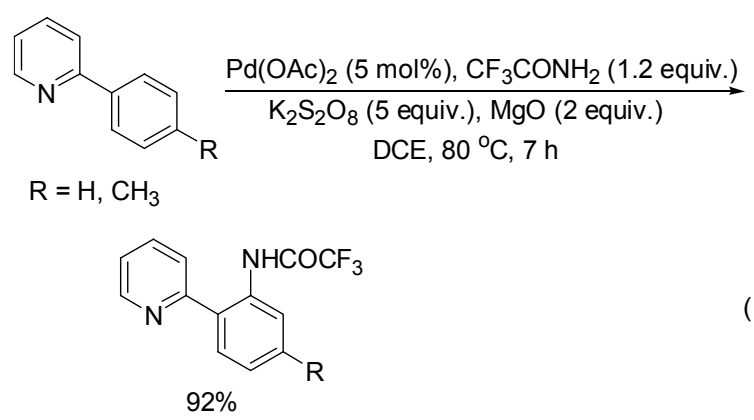

2011 年, Huang 等 ${ }^{[59]}$ 报道了氧气为氧化剂、溴化铜 催化的苯并噁唑与叔胺的分子间氧化胺化(Eq. 20). 反 应不需要外来碱的加入, 条件温和, 并且成功地将叔胺 作为芳香 $\mathrm{C}-\mathrm{N}$ 键形成反应的氮源.

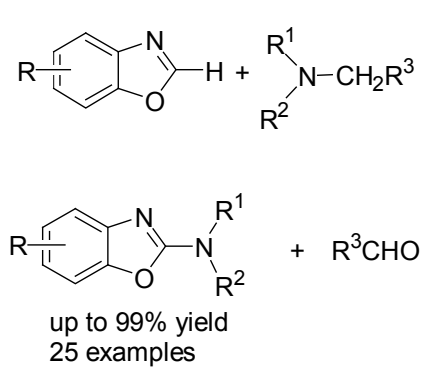




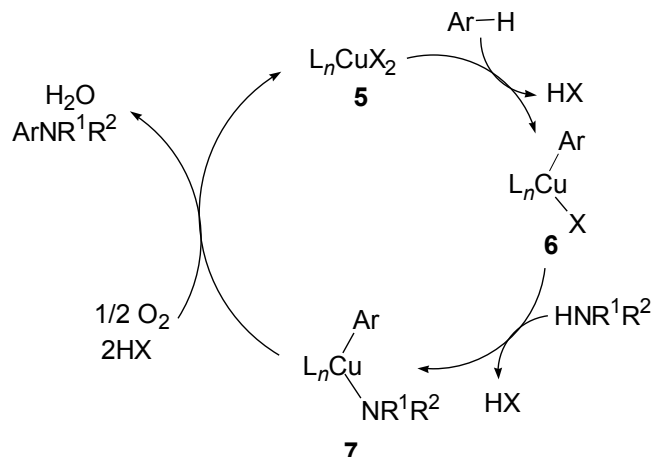

Scheme 10

$\underset{N}{\stackrel{N}{ }}$<smiles>[R]N([R])c1nc2ccccc2n1C</smiles>

$22 \% \sim 97 \%$ yields 26 examples

\section{4 传统方法的改进}

\section{1 亲核取代和亲电取代}

传统上, 芳香胺一般通过芳香 $\mathrm{C}-\mathrm{H}$ 键直接发生亲 核或者亲电取代生成 ${ }^{[64 ~ 67]}$. 近年来, 一些新的催化剂和 新的胺化试剂又丰富了该领域的内容.

2009 年, Chang 等 ${ }^{[68]}$ 报道了银诱导的甲酰胺或者胺 作为氮源的苯并噁唑的胺化 (Scheme 11). 其与甲酰胺 的反应需要在高温下进行, 而与脂肪胺的直接胺化反应 条件则相对温和. 这里, 引入光学活性胺基时也不会发 生外消旋化.

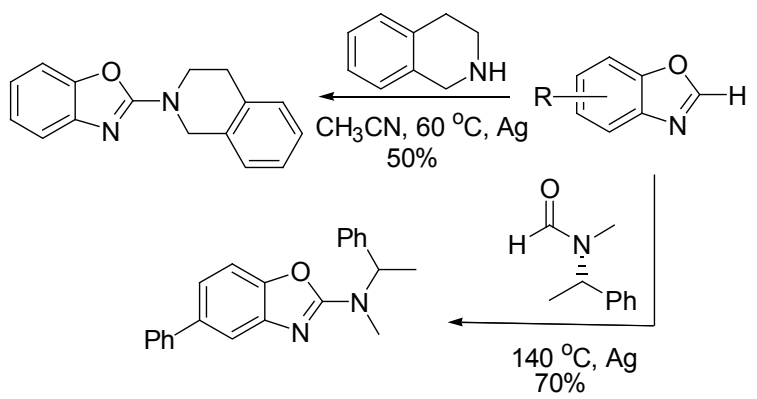

\section{Scheme 11}

2010 年, Miura 等 ${ }^{[69]}$ 报道了铜催化的氯胺作为氮源 的杂芳烃直接胺化(Eq. 25). 环状或者非环状的氯胺都 可以很容易进行该反应得到胺基取代的 1,3,4-噁二唑.

\section{2 还原环胺化}

含有 $\mathrm{C}-\mathrm{N}$ 键的氮杂环衍生物还可以通过硝基化合
物的还原环化得到 ${ }^{[70 ~ 73]}$.
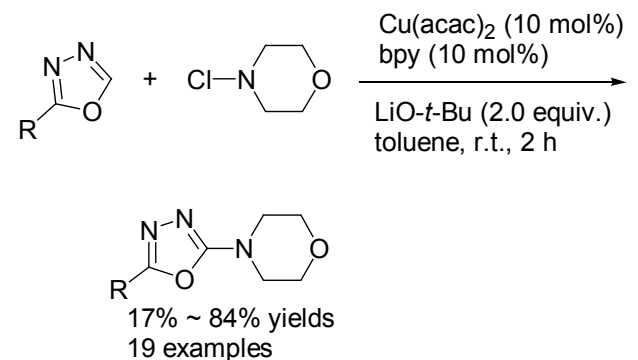

1962 年, Cadogan 等 ${ }^{[74]}$ 报道了在合适的有机膦试剂 作用下的 2-硝基联苯衍生物还原环化(Scheme 12)，这成 为咔唑常用的合成方法之一。这种方法与其他亲电合成 相比，有很多优势：拓展了底物范围，扩大了官能团兼 容性，产物里官能团位置更精确的区域控制(由联苯起 始原料的相对位置决定).
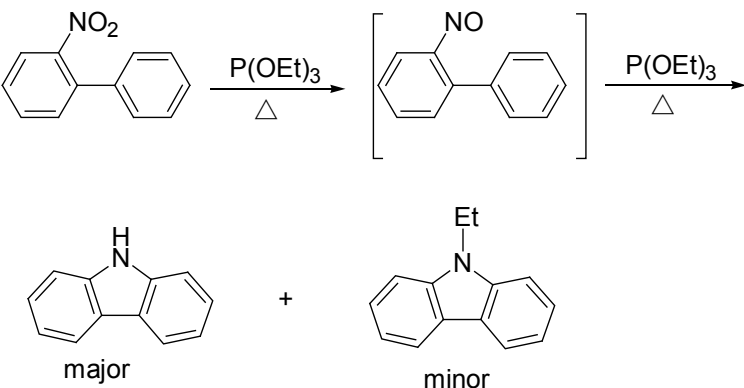

Scheme 12

2004 年, Smitrovich 等 ${ }^{[75]}$ 报道了在醋酸钯/菲的作用 下，活化芳香 $\mathrm{C}-\mathrm{H}$ 键形成分子内 $\mathrm{C}-\mathrm{N}$ 键的转化，可方 便地将 2-硝基联苯转变为咔唑(Eq. 26). 此反应机理尚 不明确，但 2-氨基联苯并非反应的中间体.<smiles>O=[N+]([O-])c1ccccc1-c1ccccc1</smiles>

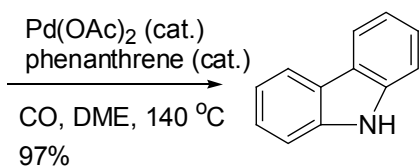

2009 年, Dong 等 ${ }^{[76]}$ 首次报道了过渡金属催化的用 一氧化碳作为末端还原剂的芳香 $\mathrm{C}-\mathrm{H}$ 键的胺化, 可由 二芳基共轭硝基烯烃生成 3-芳基吲哚(Eq. 27). 富电子 的芳香环的关环速度比缺电子的快.

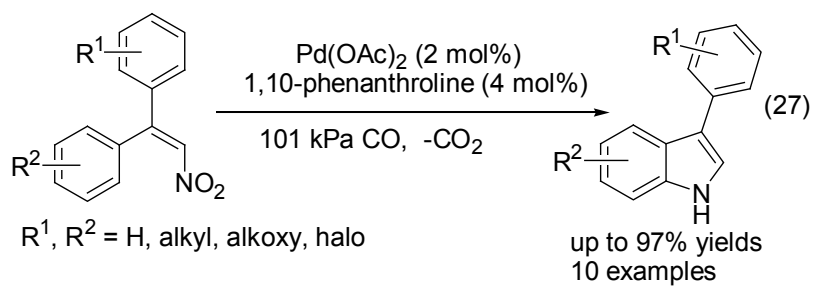




\section{5 结语}

本文对近十年来经由芳香 $\mathrm{C}-\mathrm{H}$ 键直接活化构建芳 香 $\mathrm{C}-\mathrm{N}$ 键的转化所取得的进展做了全面综述 ${ }^{[77 ~ 84]}$. 不 难看出, 该类转化经过大量化学工作者多年的探索与研 究已日檨完善 ${ }^{[85 ~ 88]}$. 不仅反应所需条件已变得更为温 和、操作更为简单, 一些绿色化反应介质如水 ${ }^{[89,90]}$ 也已 经在该反应中得到了应用, 进一步扩大了其应用范围. 尽管如此, 在这一领域中仍然存在着一些问题需进一步 改进, 如减少贵金属催化剂和配体的使用等. 䀟庸置言, 只要我们坚持努力, 这一转化在不久的将来会变得更加 实用 ${ }^{[91]}$.

\section{References}

[1] Amo, V. D.; Dubbaka, S. R.; Krasovskiy, A.; Knochel, P. Angew. Chem., Int. Ed. 2006, 45, 7838.

[2] Salomé, C.; Schmitt, M.; Bourguignon, J. J. Tetrahedron Lett. 2009, 50, 3798.

[3] Barker, T. J.; Jarvo, E. R. J. Am. Chem. Soc. 2009, 131, 15598.

[4] Hatakeyama, T.; Yoshimoto, Y.; Ghorai, S. K.; Nakamura, M. Org. Lett. 2010, 12, 1516.

[5] Guram, A. S.; Rennels, R. A.; Buchwald, S. L. Angew. Chem., Int. Ed. 1995, 34, 1348.

[6] Louie, J.; Hartwig, J. F. Tetrahedron Lett. 1995, 36, 3609.

[7] Finet, J. P.; Fedorov, A. Y.; Combes, S.; Boyer, G. Curr. Org. Chem. 2002, 6, 597.

[8] Kunz, K.; Scholz, U.; Ganzer, D. Synlett 2003, 2428.

[9] Xu, H. J.; Man, Q. S.; Lin, Y. C. Chin. J. Org. Chem. 2010, 30,9 (in Chinese).

(许华建, 蔄秋石, 林义成, 李源源, 冯乙巳, 有机化学, 2010, 30, 9.)

[10] Li, J. R.; Li, J. J. Reaction of Aromatic C $-H$ Bonds, Science Press, Beijing, 2008 (in Chinese).

(李加荣, 李建军, 芳香 $\mathrm{C}-\mathrm{H}$ 键的反应, 科学出版社, 北京, 2008.)

[11] Corbet, J. P.; Mignani, G. Chem. Rev. 2006, 106, 2651.

[12] Colby, D. A.; Bergman, R. G.; Ellman, J. A. Chem. Rev. 2010, 110, 624.

[13] Tan, Y. C.; Hartwig, J. F. J. Am. Chem. Soc. 2010, 132, 3676.

[14] For Pd: (a) Liu, G.; Yin, G.; Wu, L. Angew. Chem., Int. Ed. 2008, $47,4733$.

(b) Jiang, H. F.; Ji, X. C.; Li, Y. B.; Chen, Z. W.; Wang, A. Z. Org. Bio. Chem. 2011, 9, 5358 .

[15] For Co: Ragaini, F.; Penoni, A.; Gallo, E.; Tollari, S.; Gotti, C. L.; Lapadula, M.; Mangioni, E.; Cenini, S. Chem. Eur. J. 2003, 9, 249.

[16] For Fe: (a) Bach, T.; Schlummer, B.; Harms, K. Chem. Eur. J. 2001, 7, 2581.

(b) Srivastava, R. S. Appl. Organomet. Chem. 2006, $20,851$.

(c) Tanino, T.; Ichikawa, S.; Matsuda, A. Org. Lett. 2011, 13, 4028.

(d) Chen, G. Q.; Xu, Z. J.; Liu, Y. G.; Zhou, C. Y.; Che, C. M. Synlett 2011, 1174.

(e) Ton, T. M. U.; Tejo, C.; Tania, S.; Chang, J. W. W.; Chan, P. W. H. J. Org. Chem. 2011, 76, 4894.

[17] For Rh: (a) Espino, C. G.; Wehn, P. M.; Chow, J.; Bois, J. D. J. Am. Chem. Soc. 2001, 123, 6935.

(b) Yamawaki, M.; Tsutsui, H.; Kitagaki, S.; Anada, M.; Hashimoto, S. Tetrahedron Lett. 2002, 43, 9561. (c) Espino, C. G.; Fiori, K. W.; Kim, M.; Bois, J. D. J. Am. Chem. Soc. 2004, 126, 15378.

(d) Kim, M.; Mulcahy, J. V.; Espino, C. G.; Bois, J. D. Org. Lett. 2006, 8, 1073.

(e) Liang, C.; Robert-Peillard, F.; Fruit, C.; Miller, P.; Dodd, R. H.; Dauban, P. Angew. Chem., Int. Ed. 2006, 45, 4641.

(f) Lebel, H.; Huard, K. Org. Lett. 2007, 9, 639.

(g) Kornecki, K. P.; Berry, J. F. Chem.-Eur. J. 2011, 17, 5827.

[18] For Ru: (a) Leung, S. K.Y.; Huang, J. S.; Liang, J. L.; Che, C. M.; Zhou, Z. Y. Angew. Chem., Int. Ed. 2003, 42, 340.

(b) Fantauzzi, S.; Gallo, E.; Caselli, A.; Piangiolino, C.; Ragaini, F.; Cenini, S. Eur. J. Org. Chem. 2007, 6053.

[19] For Mn: Kohmura, Y.; Katsuki, T. Tetrahedron Lett. 2001, 42, 3339.

[20] For Cu: (a) Thomas, A. W.; Ley, S. V. Angew. Chem., Int. Ed. 2003, 42,5400 .

(b) Beletskaya, I. P.; Cheprakov, A. V. Coord. Chem. Rev. 2004, 248, 2337.

(c) Badiei, Y. M.; Dinescu, A.; Dai, X.; Palomino, R. M.; Heinemann, F. W.; Cundari, T. R.; Warren, T. H. Angew. Chem., Int. Ed. 2008, 47, 9961.

(d) Niu, M. Y.; Yin, Z. M.; Fu, H.; Jiang, Y. Y.; Zhao, Y. F. J. Org. Chem. 2008, 73, 3961.

[21] For Ag: (a) Cui, Y.; He, C. Angew. Chem., Int. Ed. 2004, 43, 4210. (b) Li, Z.; Capretto, D. A.; Rahaman, R.; He, C. Angew. Chem., Int. Ed. 2007, 46, 5184 .

[22] For Ir: (a) Fujita, K.; Nakaguma, H.; Hamada, T.; Yamaguchi, R. J. Am. Chem. Soc. 2003, 125, 12368.

(b) Tomotaka, H.; Satoshi, S.; Yasutaka, I. Adv. Synth. Catal. 2005, $347,872$.

[23] Stokes, B. J.; Dong, H.; Leslie, B. E.; Pumphrey, A. L.; Driver, T. G. J. Am. Chem. Soc. 2007, 129, 7500.

[24] Chiba, S.; Hattori, G.; Narasaka, K. Chem. Lett. 2007, 36, 52.

[25] Di'az-Requejo, M. M.; Pe'rez, P. J.; Brookhart, M.; Templeton, J. L. Organometallics 1997, 16, 4399.

[26] Caballero, A.; Di'az-Requejo, M. M.; Belderrain, T. R.; Nicasio, M. C.; Trofimenko, S.; Pérez, P. J. J. Am. Chem. Soc. 2003, 125, 1446.

[27] De'az-Requejo, M. M.; Belderrain, T. R.; Nicasio, M. C. J. Am. Chem. Soc. 2003, 125, 12078.

[28] Li, Z.; Capretto, D. A.; Rahaman, R. O.; He, C. J. Am. Chem. Soc. 2007, 129, 12058.

[29] Dick, A. R.; Remy, M. S.; Kampf, J. W.; Sanford, M. S. Organometallics 2007, 26, 1365.

[30] Lyons, T. W.; Sanford, M. S. Chem. Rev. 2010, 110, 1147.

[31] Jensen, M. P.; Lange, S. J.; Mehn, M. P.; Que, E. L.; Que, L. Jr. J. Am. Chem. Soc. 2003, 125, 2113.

[32] King, E. R.; Betley, T. A. Inorg. Chem. 2009, 48, 2361.

[33] Ng, K. H.; Chan, A. S. C.; Yu, W. Y. J. Am. Chem. Soc. 2010, 132, 12862.

[34] Ye, X.; Liu, G. S.; Popp, B. V.; Stahl, S. S. J. Org. Chem. 2011, 76, 1031.

[35] Sarkar, S. D.; Studer, A. Org. Lett. 2010, 12, 1992.

[36] Jia, W.; Jiao, N. Org. Lett. 2010, 12, 2000.

[37] Kienle, M.; Wagner, A. J.; Dunst, C.; Knochel, P. Chem.-Asian J. 2011, 6, 517 .

[38] Matsubara, S.; Yokota, Y.; Oshima, K. Chem. Lett. 2004, 33, 294.

[39] Matsubara, S.; Yokota, Y.; Oshima, K. Org. Lett. 2004, 6, 2071.

[40] Yamamoto, M.; Matsubara, S. Chem. Lett. 2007, 36, 172.

[41] Tsang, W. C. P.; Zheng, N.; Buchwald, S. L. J. Am. Chem. Soc. 2005, 127, 14560.

[42] Tsang, W. C. P.; Munday, R. H.; Brasche, G.; Zheng, N.; Buchwald, S. L. J. Org. Chem. 2008, 73, 7603.

[43] Giri, R.; Chen, X.; Yu, J. Q. Angew. Chem., Int. Ed. 2005, 44, 2112. 
[44] Wang, D. H.; Hao, X. S.; Wu, D. F.; Yu, J. Q. Org. Lett. 2006, 8, 3387.

[45] Giri, R.; Wasa, M.; Breazzano, S. P.; Yu, J. Q. Org. Lett. 2006, 8, 5685.

[46] Li, J. J.; Mei, T. S.; Yu, J. Q. Angew. Chem., Int. Ed. 2008, 47, 6452.

[47] Inamoto, K.; Saito, T.; Katsuno, M.; Sakamoto, T.; Hiroya, K. Org. Lett. 2007, 9, 2931.

[48] Wasa, M.; Yu, J. Q. J. Am. Chem. Soc. 2008, 130, 14058.

[49] Wang, D. H.; Mei, T. S.; Yu, J. Q. J. Am. Chem. Soc. 2008, 130, 17676.

[50] Jordan-Hore, J. A.; Johansson, C. C. C.; Gulias, M.; Beck, E. M.; Gaunt, M. J. J. Am. Chem. Soc. 2008, 130, 16184.

[51] Brasche, G.; Buchwald, S. L. Angew. Chem., Int. Ed. 2008, 47, 1932.

[52] Inamoto, K.; Saito, T.; Hiroya, K.; Doi, T. Synlett 2008, 3157.

[53] Mei, T.-S.; Wang, X.; Yu, J. Q. J. Am. Chem. Soc. 2009, 131, 10806.

[54] Youn, S. W.; Bihn, J. H.; Kim, B. S. Org. Lett. 2011, 13, 3738.

[55] Wunderlich, S. H.; Kienle, M.; Knochel, P. Angew. Chem., Int. Ed. 2009, 48, 7256.

[56] Kienle, M.; Dunst, C.; Knochel, P. Org. Lett. 2009, 11, 5158.

[57] Chen, X.; Hao, X. S.; Goodhue, C. E.; Yu, J. Q. J. Am. Chem. Soc. 2006, 128, 6790.

[58] Thu, H. Y.; Yu, W. Y.; Che, C. M. J. Am. Chem. Soc. 2006, 128, 9048.

[59] Guo, S. M.; Qian, B.; Xie, Y. J.; Xia, C. G.; Huang, H. M. Org Lett. 2011, 13, 522.

[60] Froehr, T.; Sindlinger, C. P.; Kloeckner, U.; Finkbeiner, P.; Nachtsheim, B. J. Org. Lett. 2011, 13, 3754.

[61] Chiba, S.; Kitamura, M.; Saku, O.; Narasaka, K. B. J. Chem. Soc. Jpn. 2004, 77, 785.

[62] Monguchi, D.; Fujiwara, T.; Furukawa, H.; Mori, A. Org. Lett. 2009, 11, 1607.

[63] Wang, Q.; Schreiber, S. L. Org. Lett.2009, 11, 5178.

[64] Wohl, A. Chem. Ber. 1903, 36, 4135.

[65] Becker, J.; Holderich, W. F. Catal. Lett. 1998, 54, 125.

[66] Storz, T. Org. Proc. Res. Dev. 2004, 8, 663.
[67] Chakrabarty, M.; Rhosh, N.; Harigaya, Y. Heterocycles 2004, 62, 779.

[68] Cho, S. H.; Kim, J. Y.; Lee, S. Y.; Chang, S. Angew. Chem., Int. Ed . 2009, 48, 9127.

[69] Kawano, T.; Hirano, K.; Satoh, T.; Miura, M. J. Am. Chem. Soc. 2010, 132, 6900.

[70] Cadogan, J. I. G. Quart. Rev. 1962, 16, 208.

[71] Freeman, A. W.; Urvoy, M.; Criswell, M. E. J. Org. Chem. 2005 $70,5014$.

[72] Smitrovich, J. H.; Davies, I. W. Org. Lett. 2004, 6, 533.

[73] Wolfe, J. P.; Thomas, J. S. Curr. Org. Chem. 2005, 9, 625.

[74] Cadogan, J. J. G. Quart Rev. 1962, 16, 208.

[75] Smitrovich, J. H.; Davies, J. W. Org. Lett. 2004, 6, 533.

[76] Hsich, T. H.; Dong, V. M. Tetrahedron 2009, 65, 3062.

[77] Derouane, E. G. Cattech 2001, 5, 214.

[78] Labinger, J. A.; Bercaw, J. E. Nature 2002, 417,507.

[79] Periana, R. A.; Mironov, O.; Taube, D.; Bhalla, G.; Jones, C. J. Science 2003, 301, 814 .

[80] Davies, H. M. L.; Manning, J. R. Nature 2008, 451,417.

[81] Phipps, R. J.; Gaunt, M. J.; Affiliations, A. Science 2009, 323, 1593.

[82] Wang, D. H.; Engle, K. M.; Shi, B. F.; Yu, J. Q. Science 2010, 327, 315.

[83] Lu, H. J.; Zhang, X. P. Chem. Soc. Rev. 2011, 40, 1899.

[84] Collet, F.; Lescot, C.; Dauban, P. Chem. Soc. Rev. 2011, 40, 1926.

[85] Muller, K.; Faeh, C.; Diederich, F. Science 2007, 317, 188.

[86] Ueda, S.; Nagasawa, H. Angew. Chem., Int. Ed. 2008, 47, 6411.

[87] Chen, X.; Dobereiner, G.; Hao, X. S.; Giri, R.; Maugel, N.; Yu, J. Q. Tetrahedron 2009, 65, 3085

[88] Zhao, D.; Wang, W.; Yang, F.; Lan, J.; Yang, L.; Gao, G.; You, J. Angew. Chem., Int. Ed. 2009, 48, 3296.

[89] Baslé, O.; Li, C. J. Green Chem. 2007, 9, 1047.

[90] Demidov, O. P.; Borovlev, I. V.; Saigakova, N. A.; Nemykina, O. A.; Demidova, N. V.; Pisarenko, S. V. Chem. Heterocycl. Compd. 2011, 47, 114.

[91] Wang, Y. F.; Zeng, J. H.; Cui, X. R. Chin. J. Org. Chem. 2010, 30, 181 (in Chinese)

（王晔峰，曾京辉，崔晓瑞，有机化学，2010,30,181.）

(Qin, X.) 